\title{
Doppelte Kompetenz für den digitalen Wandel
}

Thieme und m.Doc schlagen ein wegweisendes Kapitel im patientenorientierten Informationsmanagement auf. Durch die integrierte Partnerlösung gelingt es dem Patienten einfacher denn je, zeit- und ortsunabhängig relevante Inhalte und Services aufzurufen.

Patienten haben es manchmal nicht leicht. Verletzungen oder Krankheiten bereiten schon genug Sorgen. Getoppt wird das Übel durch das Gefühl, mit all den Unsicherheiten, die ein Krankenhausaufenthalt impliziert, überfordert zu sein. Wer hilft mir schnell und verständlich weiter, wenn ich mich frage: „Habe ich an alles gedacht?“ „Was passiert heute mit mir?“ „Wie geht es jetzt mit mir weiter?“ Doch auch auf Klinikseite wünscht man sich reibungslose Abläufe. Der Patient soll seine Unterlagen bitte vollständig abgeben, natürlich lückenlos ausgefüllt. Und an seine Therapietermine möchte man ihn nicht in letzter Sekunde erinnern müssen - selbst ist der Patient, hundertprozentig zuverlässig, idealerweise. Um der stockenden Realität einen lebendigen Informationsfluss zwischen Patienten, Praxen, Kliniken und Kostenträgern entgegenzusetzen, ist ein digitales Umdenken nötig. Werden innovative Anwendungen genutzt und Schnittstellen zwischen allen Beteiligten geschaffen, ist der Weg frei für eine maximal fokussierte Patientenkommunikation und -behandlung; ohne dicke Mappen, verlegte Formulare, doppelte Untersuchungen oder versäumte Therapiestunden. Geld und Zeit werden gespart, Nerven geschont.

Der Idee, den Datenfluss zwischen medizinischen Einrichtungen und Patienten zu verbessern, haben sich die m.Doc GmbH und die Thieme Gruppe angenommen. Das Kernprodukt des Kölner Unternehmens m.Doc ist eine digitale Gesundheitsplattform, auf welcher u.a. die Lösung „Smart Clinic“ betrieben wird. Mit Smart Clinic erhält der Patient alle nötigen Informationen für die Zeit vor, während und nach seinem Klinikaufent-

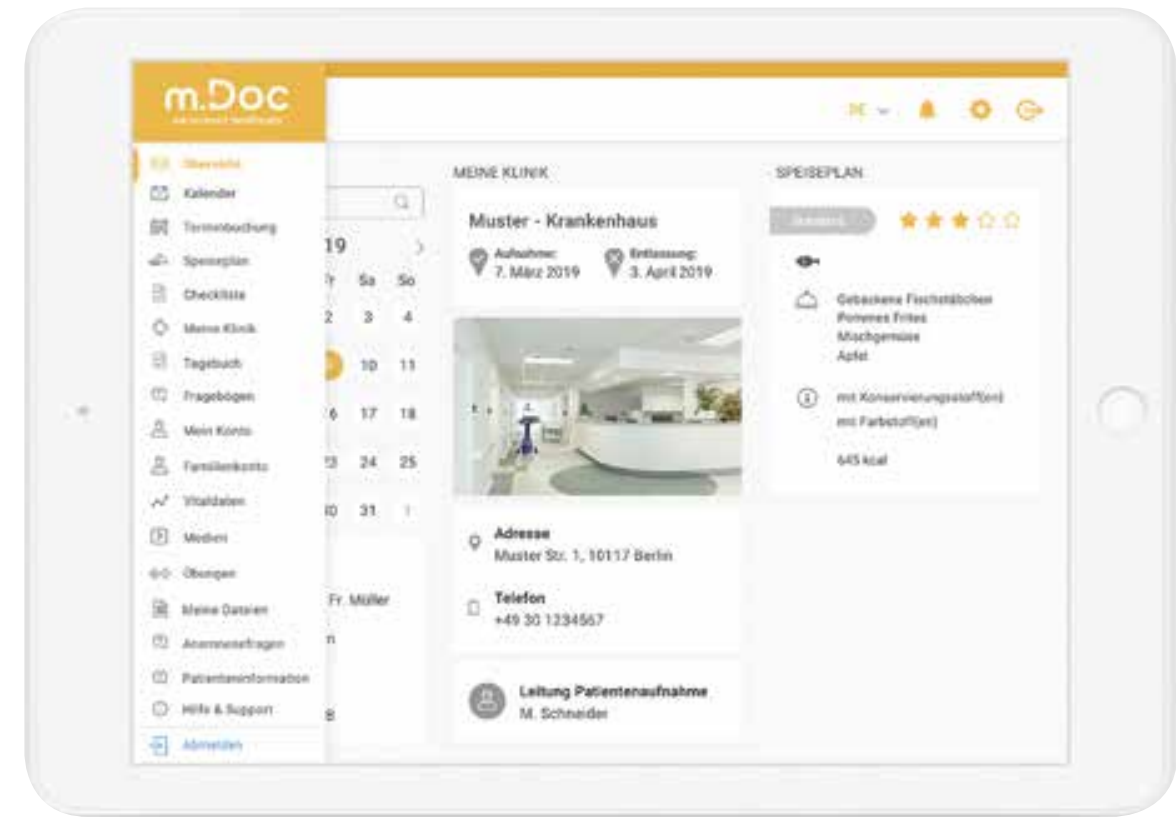

Mit Smart Clinic erhält der Patient alle nötigen Informationen für die Zeit vor, während und nach seinem Klinikaufenthalt. Foto: m.Doc

halt - vom Aufnahmeprocedere bis zum Follow-Up-Training. Angebunden ist die App an Krankenhausinformationssysteme, elektronische Patientenakten und qualifizierte Anwendungen - das Partnernetzwerk wächst stetig an. Das Resultat: Effiziente Informationsprozesse, die dem Patienten das Leben leichter machen - und dem medizinischen Personal; denn wenn unnötige Fragen und lästige Bürokratie entfallen, profitiert das interne Klima - die Behandlung kann mit ruhiger Hand und der nötigen Konzentration durchgeführt werden. Thieme liegt mit seiner Mission auf einer ähnlichen Linie: „Mit den entscheidenden Informationen zur richtigen Zeit am richtigen Ort.“ Die Gruppe bietet ein umfassendes Spektrum an Produkten zur Optimierung von Informationsprozessen zwischen Patienten, Leistungserbringern und Kostenträgern. Beispiele sind die Software E-ConsentPro, eine ganzheitliche Lösung für medizinisch und juristisch fundierte Patientenaufklärung, oder die anschaulichen Aufklärungsfilme zu verschiedensten operativen Eingriffen. Durch die Kooperation der beiden Unternehmen und der damit verbundenen Integration von Thieme Content in die Software Smart Clinic wird für den Patienten ein deutlicher Mehrwert geschaffen.

Wie die Verknüpfung von Thieme Angeboten und Smart Clinic von m.Doc konkret aussehen kann, zeigt beispielhaft Emmas Patientenreise:

\section{Emma bekommt einen Termin}

Emma ist 56 Jahre alt und liebt ihren Garten. Leider kann sie dort nicht mehr so 


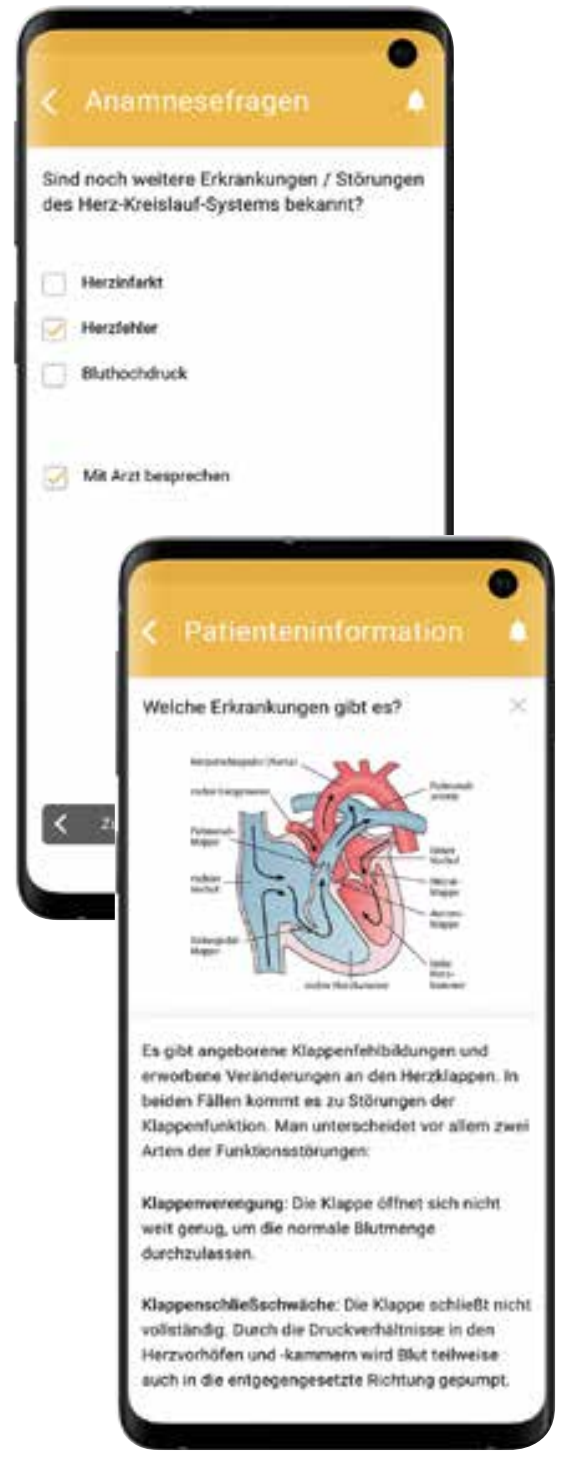

(o.) Integrierte Partnerlösung: Die Anamnesefragen aus dem Hause Thieme Compliance kann Emma über die m.Doc Plattform mit Hilfe der Smart Clinic App beantworten ...

(r.) ... und die Informationstexte zur Herz-OP lesen. Fotos: m.Doc

aktiv sein, da sich ihre Herzinsuffizienz verschlechtert hat. Deswegen rät ihr Arzt zu einer Herzklappen-Operation; er empfiehlt ihr ein Krankenhaus in ihrer Nähe. Der Arzt gibt ihr außerdem den Tipp, sich die App Smart Clinic vom lokalen Krankenhaus auf ihr Handy zu laden. Und genau das macht Emma.

Emma ist überrascht. Für die erste ambulante Vorbesprechung kann sie über die App direkt einen Termin in der Klinik buchen. Sie wählt den kommenden Mitt-
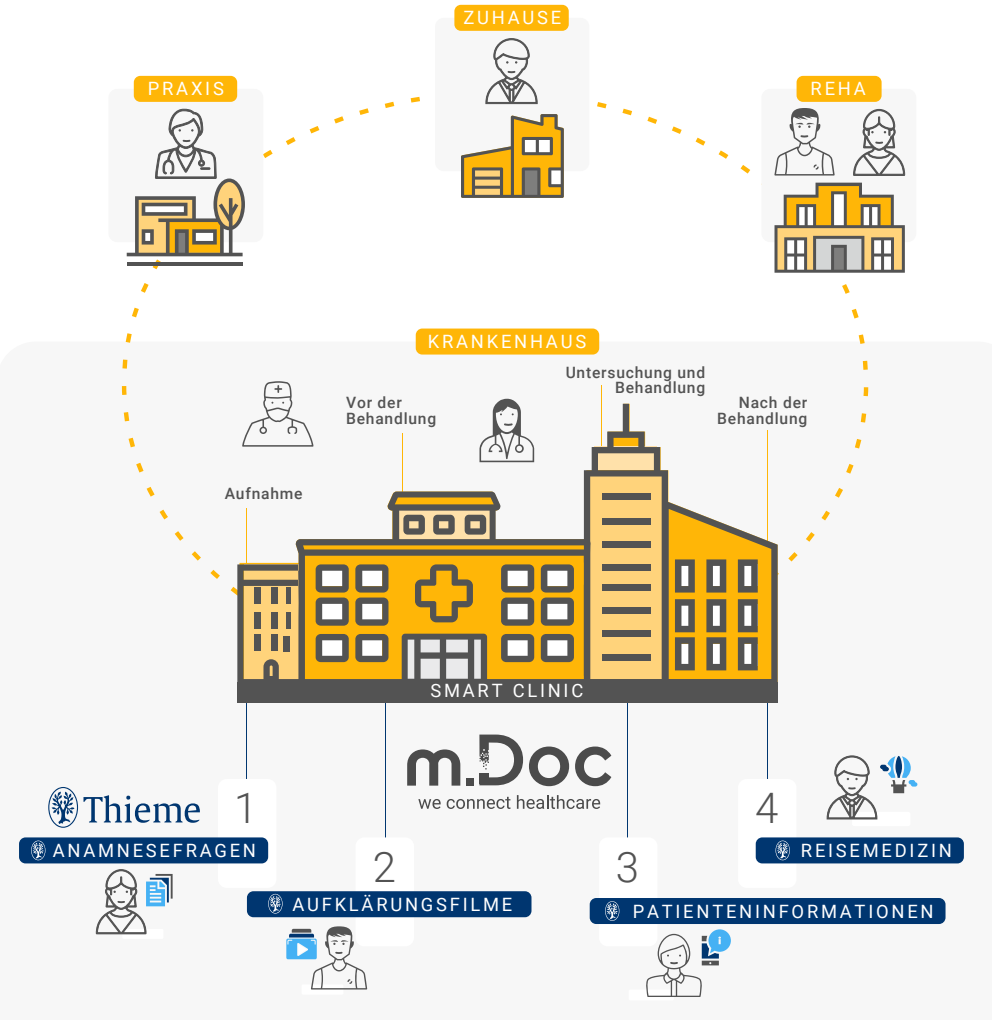

m.Doc | DIGITALE GESUNDHEITSPLATTFORM

Entlang der Patient Journey: Einbindung von Thieme Content in die Gesundheitsplattform Smart Clinic. Illustration: m.Doc

woch. Die Klinik bestätigt ihr den Termin in kürzester Zeit. Zugleich entdeckt Emma in der App einen virtuellen Thieme Anamnesebogen, den sie zu Hause ausfüllt und eine klinikspezifische Checkliste mit den Punkten, an die sie denken soll. Dem Wunsch, Überweisung und Brief vom Facharzt zu übersenden, kommt sie bequem per App nach. Für die Datensicherheit ist auch gesorgt: Emma wird aufgefordert, ein Passwort zu wählen und zweimal zu bestätigen. Geschafft. Moment, da war doch noch etwas. Emma loggt sich erneut ein und ruft einen Film auf, der ihr Sicherheitshinweise und Informationen für den bevorstehenden Klinikaufenthalt vermittelt. Sie staunt und fühlt sich bestens unterrichtet.

\section{Emma - und es geht los}

Emma geht für die Voruntersuchungen in die Klinik. Der Termin für die Operation wird festgelegt. Wieder zuhause findet sie in ihrer App weitere Aufklärungsbögen diesmal speziell für den Eingriff -, ein
Informationsblatt und ein Aufklärungsvideo zur anstehenden Herz-OP. Durch die anschaulichen 3-D-Animationen kann sie die ablaufenden Schritte gut nachvollziehen. Sie ist beruhigt. Noch ein Tag, dann ist es soweit. Emma geht die virtuelle Packliste Zeile für Zeile durch. Die Zahnbürste noch, der Schlafanzug und schon ist der Koffer voll. Perfekt. Emma erhält eine Erinnerung mit der genauen Uhrzeit und der Bitte, die Aufnahme-Checkliste nochmals zu prüfen.

\section{Im Krankenhaus}

Alle Informationen zu Aufenthalt und Behandlung hat sie digital zur Verfügung. Am Tag vor der Operation schaut sie sich nochmal den Aufklärungsbogen und das Video an. Das gibt ihr Sicherheit. Sie liest sich durch, worauf sie vor und nach der Operation achten muss. Ihr Schlaf ist tief und fest. Emma ist erleichtert: Die Operation verlief perfekt. Zwei Tage später geht sie die ersten Schritte und sucht sich per App ihr Essen aus - Spinat, Kartoffelbrei 
und Spiegeleier. Sie blättert per App in ihrer Lieblingszeitung und probiert die Indoor-Navigation aus, um die Cafeteria zu finden.

Emma - geschafft, und es geht nach Hause

Emma informiert sich, wie sie sich verhalten soll, damit der Genesungsprozess optimal verläuft. Immer wieder interessiert sie sich rückblickend für Details zum Eingriff. Sie plant schon länger, im Herbst nach Asien zu reisen; mit dem Informationsangebot von CRM (Centrum für Reisemedizin der Thieme Gruppe) macht sie sich schlau, was sie hierbei beachten muss. Danach füllt sie einen Fragebogen der Klinik aus. Die Daten nutzt die Klinikleitung für die Qualitätssteuerung. Am Tag ihrer Entlassung erhält Emma ihre Behandlungsunterlagen, die sie jederzeit online aufrufen kann; auch ihr Hausarzt bekommt diese Informationen. Selbst nach der Entlassung kann Emma offene Fragen klären und ihre Nachsorge wird durch gezielte Empfehlungen und Unterstützungsangebote begleitet.

Eine „Reise“ wie Emma wünscht sich jeder Patient. Die Sorgen werden auf ein Minimum reduziert, die Zuversicht auf ein Maximum erhöht. Durch die schnelle Übermittlung relevanter Informationen auf digitalem Wege wird auch das Klinikpersonal entlastet. Ein positiver Kreislauf setzt sich in Gang - und es bleibt mehr Zeit für die wirklich wichtigen Dinge.

\section{Dr. André Gärisch}

Journalist

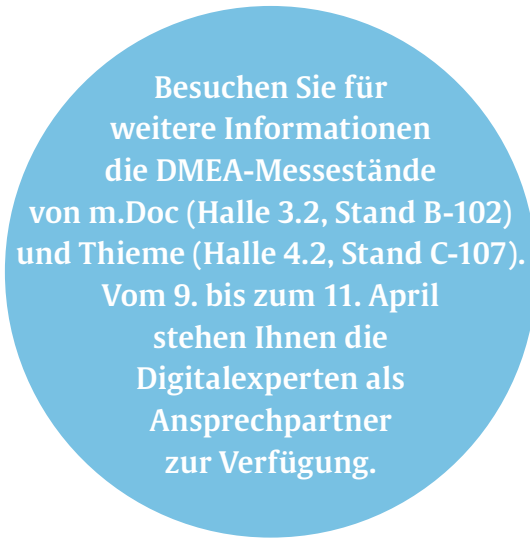

\section{Den digitalen Traum gemeinsam denken}

Für eine spürbar effiziente Digitalisierung ist entscheidend, relevante Arbeitsabläufe zu identifizieren und aus diesen Potentialen innovative Lösungen zu entwickeln.

Wer in Dokumentation und Archivierung digital denkt, entlastet Mitarbeiter und gibt dem Patienten das Gefühl, in kompetenten Händen zu sein. Nicht immer ist aber eine „Digitalisierung um jeden Preis“ das Mittel der Wahl für die papierlose Arztpraxis. Entscheidend ist vielmehr, die Arbeitsabläufe zu identifizieren, die durch eine Digitalisierung spürbar effizienter gestaltet werden können. Um diesen Potentialen des papierlosen Workflows innovative Wege zu ebnen, arbeiten Thieme Compliance und mediDOK bereits seit 2015 erfolgreich zusammen.

Enge Terminpläne, sensible Gespräche und komplexe Eingriffe prägen den Alltag im Gesundheitssektor; idealerweise greift jedes Rädchen ins andere. Trotzdem stapeln sich in Arztpraxen und Krankenhäusern immer noch Formulare auf den Schreibtischen und Ordner in den Schränken eine dokumentarische Vorgehensweise, die den Verlust von Zeit und Unterlagen begünstigt; schnell greifen Hektik und Unsicherheit um sich.

Besonders in der Patientenaufnahme und -aufklärung bieten mobile Anwendungen entscheidende Vorteile. Sämtliche Abläufe, von der Dokumentation bis zur Speicherung, sind über ein smartes Endgerät steuerbar. Daten können zentral gesammelt, gesichert und jederzeit abgerufen werden. Das gesamte System, dessen Mitarbeiter und nicht zuletzt die Patienten „atmen befreit durch“. Der Mission effizienterer Abläufe durch digitale Anwendungen haben sich Thieme Compliance, Spezialist für Patienten- aufklärung, und die mediDOK Software Entwicklungsgesellschaft, renommierter Archivlösungsanbieter, verschrieben. Seit 2015 stimmen beide Unternehmen ihre Produkte E-ConsentPro mobile und mediDOK so aufeinander ab, dass Arztpraxen und medizinischen Versorgungszentren (MVZ) ein papierloser Workflow ermöglicht wird - von der Patientenaufnahme und -aufklärung bis hin zur Datenarchivierung.

\section{Mehr Zeit für wirklich wichtige Dinge} Der Patient wünscht sich eine verständliche und sorgfältige Aufklärung. Digitale Anwendungen wie die tabletbasierte Lösung E-ConsentPro mobile bieten hier eine zielgerichtete Unterstützung. Patienten beantworten die Anamnesefragen direkt vor dem Arzt-Patienten-Gespräch auf dem Tablet - so erhält der Arzt vorab einen Einblick zum Patienten-Risikoprofil. Handschriftliche Bemerkungen und Hervorhebungen sind ebenso möglich wie zeichnerische Ergänzungen in Bildern - alles mobil. Arzt und Patient unterschreiben anschließend digital. Die signierten Aufklärungsbögen können dank der Schnittstelle zwischen E-ConsentPro mobile und mediDOK bequem und automatisch archiviert werden. Im letzten Schritt druckt der Anwender die Kopie für den Patienten aus.

Die mediDOK-Archivlösung ist in nahezu alle Patientenverwaltungssysteme integrierbar; so ist es möglich, die archivierten Aufklärungsbögen direkt beim Patienten in mediDOK zu speichern und in der elektronischen Karteikarte des 